\section{ABS84: Tuberculosis in Turkey}

Mustafa Kemal Alimoglu

Akdeniz University Faculty of Medicine, Antalya, 07059, Turkey

Introduction: According to WHO reports, one-third of the world's population is currently infected with the tuberculosis (TB) bacilli and $5-10 \%$ of them are estimated to become sick or infectious during their lives. Each year, nearly 8 million new cases appear and about 2 million people die from TB. WHO statistics are prepared on the basis of data obtained from national institutions which report only the registered cases. However, the data from countries lacking high-quality registration and communication structures in their health systems may underestimate the disease burden. Aims and objectives: The aim was to provide data about TB in Turkey. Methods: Official statistics and published or presented research reports estimating prevalence, incidence, mortality and treatment facts of TB were reviewed. Results: Although university or specialty hospitals are involved in TB management, official surveillance data are collected by a total of 272 TB dispensaries working under the Department of TB in the Ministry of Health. There are some studies showing that $18-60 \%$ of known cases are not registered in TB Dispensaries. Consequently, official prevalence $(30-40$ per 100,000$)$ and incidence rates $(24$ cases per 100,000 ) are thought to underestimate the actual condition. About 15 million people are estimated to be infected by TB bacilli and $40 \%$ of smear positive cases can be notified. Treatment is successful in $33-36 \%$ of cases. The proportions of defaulted patients in the first and recurrent therapies are $12 \%$ and 23\% respectively. Multidrug-resistant TB is prevalent in 3-5\% of new and $20-30 \%$ of old cases. Directly observed treatment strategy (DOTS) has been conducted in 5 pilot regions since 2003 and will be implemented nationwide after 2006. Conclusion: TB is still a big public health problem, but underestimated by official bodies due to lack of a working registration and communication structure in health system. Involvement of primary care in TB management and DNTS activitis! in: yelp reveal more smear positive case a d cecress rumber of defaulted and multidrug re:istant cases.

Conflict of interest and funding None.

doi:10.1016/j.pcrj.2006.04.176

ABS85: Smoking among Turkish adolescents: A review of the national literature

Emine Efe, Selma Oncel, Zeynep Canli Ozer, Mustafa Kemal Alimoglu

\section{Akdeniz University School of Health, 07058, Turkey}

Introduction: Tobacco epidemic is one of the most important public health problems all around the world. Regarding the low success rates in smoking cessation activities, it seems more reasonable to prevent starting to smoke. Adolescents have the highest risk to start smoking especially in developing countries like Turkey. Nearly $30 \%$ of Turkish population consists of young individuals between 10 to 25 years old. Since many of the studies conducted among Turkish adolescents to determine their smoking behaviour were published in local publications, sufficient data on this issue is not available in international arena. Aims and objectives: The aim was to provide data about smoking behaviour among Turkish adolescents by reviewing papers published in local journals and books. Methods: Results of 42 studies conducted between 1988 and 2005 among young people aged $12-20$ years were reviewed to determine smoking prevalence, incidence, gender distribution of smokers, starting age to smoke and smoking behaviours. Results: Mean starting age to smoke was reported between 12.6 and 16.9 years. Prevalence of regular smokers was between $1.7 \%$ and $55.9 \%$ and increasing with age. Smoking was also more prevalent in working adolescents than that of school attendants. The ratio of smokers among males was 1.1-6.3 times higher than that of females. Close friends, desire to imitate adults and curiosity were found effective on starting to smoke. In a study conducted among $12-13$ years old students, $84 \%$ of the participants reported negative feelings about smoking people; however, $92 \%$ intended to start smoking in the future although majority of them reported that they had been already informed about the harmful effects of smoking on health. Conclusions: Smoking is prevalent among Turkish adolescents. Information about smoking behaviours, starting age and reasons for smoking in young population will direct the struggle against smoking.

Conflict of interest and funding None.

doi:10.1016/j.pcrj.2006.04.177

ABS86: Smoking among health professionals: A review of the national literature

Selma Oncel, Zeynep Canli Ozer, Emine Efe, Mustafa Kemal Alimoglu

Akdeniz University Antalya School of Health, Antalya, 07058, Turkey

Introduction: Each year nearly 4.5 million people die from smoking related health problems in the world. Annual number of the people dying from the same reason is about 35000 in Turkey in which smoking is a common habit and an important public health problem. Ty-kev is the second only to Greece among European countries in cigare te consumption per person. More "than 260 c C.0 Deatin professionals work in different parts of healti system in Turkey and they have a signi ic: nt responsibility in antismoking activities. The simplest Thing to do is adophing a smoke-free life style to be a good role mo iel for he reuple around. Aims and objectives: The in of this review was to provide data about smoking teliav our among Turkish health professionals. Methods: Results of 44 studies conducted between 1981 and 2004 among health professionals and published in national journals were reviewed to determine smoking prevalence among health professionals. Results: Smoking prevalence among physicians was reported 29.0 to $59.0 \%$ (39.3-59.1\% in males and $24.9-41.0 \%$ in females). The ratio of the smokers among academicians in medical schools was 39.0 to $56.0 \%$. Of the research assistants in medical faculties, 44.0 to $57.0 \%$ were reported as active smokers. Prevalence of tobacco use was 33.3 to $58.9 \%$ among dentists. Smoking was also common among nurses with prevalence values between 43 and 59\%. Health officers and the other health workers had a smoking prevalence between 43.7 and $57.4 \%$. Frequency of smokers among health professionals has not changed significantly since 1981 (56\% in 1981 and 54\% in 2004). Conclusions: Smoking prevalence among health professionals, who are well aware of the harmful effects of tobacco on health, seems to be higher than that of general adult population. Health workers should be considered as a target population and priority should be given them in smoking cessation activities because of their vital role in the struggle against tobacco.

Conflict of interest and funding None.

doi:10.1016/j.pcrj.2006.04.178 